Research Paper

\title{
Serum Pre-Albumin Predicts the Clinical Outcome in Metastatic Castration-Resistant Prostate Cancer Patients Treated With Abiraterone
}

\author{
Liancheng Fan ${ }^{1 *}$, Chenfei Chi ${ }^{*}$, Sanwei Guo ${ }^{2 *}$, Yanqing Wang ${ }^{1}$, Wen Cai ${ }^{1}$, Xiaoguang Shao ${ }^{1}$, Fan $\mathrm{Xu}^{1}$, \\ Jiahua Pan ${ }^{1}$, Yinjie Zhu ${ }^{1}$, Xun Shangguan ${ }^{1}$, Zhixiang Xin ${ }^{1}$, Jianian Hu${ }^{1}$, Hongyang Qian ${ }^{1}$, Shaowei Xie ${ }^{1}$, Rui \\ Wang ${ }^{3}$, Lixin Zhou ${ }^{1}$, Baijun Dong ${ }^{1 凶}$, Wei Xue ${ }^{1 凶}$ \\ 1. Department of Urology, Renji Hospital, School of Medicine, Shanghai Jiao Tong University, Shanghai 200127,China; \\ 2. Department of Urology, Shanghai International Medical Center, No.4358 KangXin Awy, Shanghai 200000,China; \\ 3. Shanghai Institute of Ultrasound in Medicine, Department of Ultrasonography, Shanghai Jiaotong University Affiliated No. 6 Hospital, Yishan Road 600, \\ Shanghai 200233, PR China. \\ * Liancheng Fan, Chenfei Chi and Sanwei Guo contributed equally. \\ $\square$ Corresponding authors: Baijun Dong and Wei Xue, Department of Urology, Renji Hospital, School of Medicine, Shanghai Jiao Tong University, Shanghai \\ 200127, China E-mail addresses: dongbaijun@renji.com (Dong, B) xuewei@renji.com (Xue, W). \\ ( $)$ Ivyspring International Publisher. This is an open access article distributed under the terms of the Creative Commons Attribution (CC BY-NC) license \\ (https://creativecommons.org/licenses/by-nc/4.0/). See http://ivyspring.com/terms for full terms and conditions.
}

Received: 2017.05.22; Accepted: 2017.08.31; Published: 2017.09.20

\begin{abstract}
Objective To determine the prognostic utility of serum pre-albumin in metastatic castration-resistant prostate cancer (mCRPC) patients treated with abiraterone (AA).

Patients and Methods 112 chemotherapy pretreated or chemotherapy-naive patients were scheduled for systemic treatment with AA. Serum pre-albumin levels were measured before and after 3 months of AA treatment. Univariate and multivariate analyses were performed to determine prognostic factors that were associated with PSA progression-free survival (PSA-PFS), radiographic PFS (rPFS) and overall survival (OS). The Harrell concordance index with variables only or combined pre-albumin data were used to evaluate the prognostic accuracy.

Results The group of patients with baseline pre-albumin value $\geq 20 \mathrm{mg} / \mathrm{dL}$ had a longer OS, PSA-PFS, rPFS than those with pre-albumin value $<20 \mathrm{mg} / \mathrm{dL}$. Based on the values of pre-albumin before and after 3 months of AA treatment, we divided these patients into 4 groups: high-high, high-low, low-high and low-low group. High- high group showed a significantly better OS, PSA-PFS, rPFS than other 3 groups. In multivariate analysis, low pre-albumin level remained significant predictors of $O S(H R, 13.2 ; P<0.001)$, rPFS (HR, 3.7; $\mathrm{P}=0.003)$ and PSA-PFS $(H R, 8.7 ; \mathrm{P}<0.001)$. The estimated $\mathrm{c}$-index of the multivariate model for OS increased from 0.814 without pre-albumin to 0.845 when pre-albumin added.

Conclusion Low pretreatment serum pre-albumin is a negative independent prognosticator of survival outcomes in $\mathrm{mCRPC}$ treated with AA and also increases the accuracy of established prognostic model. Serial pre-albumin evaluation might help clinicians guide clinical treatment of $m C R P C$ patients.
\end{abstract}

Key words: Metastatic castration-resistant prostate cancer, abiraterone acetate, prognostic model, nutrition, pre-albumin.

\section{Introduction}

Prostate cancer (PCA) is the most common cancer in men worldwide currently [1]. When it becomes metastatic castration-resistant prostate cancer (mCRPC), it is a heterogeneous disease with enormous variability of prognosis and treatment response between patients. Currently how to predict treatment response and prognosticate in these patients poses a major challenge. With the development of new agents treating MCRPC patients [2-11], how to better select treatment and sequencing 
of these drugs is increasingly investigated. Owing to these, it is crucial to identify prognostic factors of these drugs, which would allow for the assessment of individual risk profiles.

Recent studies indicate that the prognosis of cancer is associated not only with the tumor cellular differentiation and biological behavior, but also with the immunological and nutritional status $[12,13]$. The effect of inflammation in prognosis and progression has been shown in mCRPC patients. For example, neutrophil-to-lymphocyte ratio (NLR) has shown a prognostic role in $\mathrm{mCRPC}$ patients also when treated with abiraterone (AA), enzalutamide, docetaxel, or cabazitaxel [14-17]. Nevertheless, the potential prognostic pretreatment factors are still limited in $\mathrm{mCRPC}$. The nutritional status of patients with cancer is an important parameter affecting survival outcomes as well [18], while there are few studies who evaluate the prognostic factor of nutritional index in mCRPC. Fiala et al. found that serum albumin level, which is commonly used to assess the nutritional status, is an important prognostic factor in advanced non-small cell lung cancer [19]. Rosa et al showed lower pre-treatment level of albumin was associated with shorter PFS and OS of mCRPC treated with AA or enzalutamide [20]. Owing to these, we hypothesized that nutritional status may also play an important role of in the prognosis of $\mathrm{mCRPC}$.

Pre-albumin, which is a sensitive marker for determining the state of malnutrition and easily quantified in hospital laboratories, is a visceral liver-synthesized protein. Studies have shown that pre-albumin has been proven to be a significant prognostic factor for upper tract urothelial [21], renal cancer [22], esophagus [23], and lung cancers [24] et al. However, the prognostic value of serum pre-albumin levels for PCA has not yet been reported.

Thus, this study was designed to clarify the potential prognostic value of pretreatment pre-albumin and albumin level in mCRPC patients treated with AA. Moreover, for enhancing the predictive accuracy for the prognosis of $\mathrm{mCRPC}$ patients treated with AA, we develop a prognostic model for these patients.

\section{Patients and Methods}

\section{Patients}

After obtaining the approval from the Committee for Ethics of Renji Hospital and informed consents of patients, 112 chemotherapy pretreated or chemotherapy-naive patients were scheduled for systemic treatment with AA. Patients were treated at the Department of Urology, Renji hospital between 2012 and 2016. Men aged $\geq 18$ years with $\mathrm{mCRPC}$ were eligible: histologically confirmed adenocarcinoma of the prostate; ongoing ADT with a serum testosterone level of less than $50 \mathrm{ng} / \mathrm{dL}(1.7 \mathrm{nmol} / \mathrm{L})$; previous anti-androgen therapy followed by documented PSA progression after discontinuing the anti-androgen, disease progression according to Prostate Cancer Clinical Trials Working Group 2 (PCWG2) criteria.

Clinical and pathological characteristics on age, serum prostate-specific antigen (PSA) level, serum albumin and pre-albumin level and blood cell counts, biopsy Gleason score were collected. All blood samples were measured before AA treatment and serum albumin and pre-albumin levels were also measured after 3 months of AA treatment.

The study endpoints were PSA progressive free survival (PSA-PFS), radiographic progressive free survival (rPFS) and overall survival (OS). PSA-PFS was according to PCWG2 criteria [25], and radiographic progression was based on Response Evaluation Criteria in Solid Tumors (RECIST) [25] or two or more new bone lesions on bone scan or death, whichever occurred first.

\section{Laboratory assays}

Serum pre-albumin levels and other blood samples investigations were measured 1 week within before AA treatment and serum albumin and pre-albumin levels were also measured after 3 months of AA treatment. The serum pre-albumin level was assessed using turbidmetric immunoassay (Hitachi, Tokyo, Japan).

\section{Statistical Analysis}

The cutoff points to stratify pre-albumin and albumin were using pre-albumin $<20 \mathrm{mg} / \mathrm{dl}$ to identify low pre-albumin, albumin $<35 \mathrm{~g} / \mathrm{L}$ to identify hypoalbuminemia [26]. The cutoff points to stratify lactic dehydrogenase (LDH) and PSA was using LDH and PSA $\geq$ median to identify high LDH and PSA. The optimal cut-off value for classifying NLR as low or high for subsequent analysis, which was determined using ROC curve analysis, was 3.

Frequencies and proportions were assessed for categorical variables, whereas means, medians, and ranges were computed for continuous variables. For categorical variables, Pearson's chi-squared test was used. Correlations with PSA-PFS, and rPFS, and OS were assessed using Kaplan-Meier curves with log-rank statistics. Furthermore, uni- and multivariate Cox regression analyses were used to calculate their respective hazard ratios (HRs) and 95\% CIs. Only factors significant in univariate analyses were included in the subsequent multivariate analyses. A test result was considered as statistically significant for $\mathrm{P}<0.05$. The $\mathrm{c}$-index was built based on training set 
with the R package 'survival. We used SPSS version 21.0 for other key analyses.

\section{Results}

\section{Patients Characteristics}

A total of 112 mCRPC patients met eligibility criteria between 2012 and 2016. The clinical and pathological data of all patients are listed in Table 1. $42(37.5 \%)$ patients had received chemotherapy before AA treatment, while the others not. There was no significant heterogeneity between chemotherapynaïve and post- chemotherapy setting in this study (Table 2). Median age was 72 years (66 77). At enrolment $112(100 \%)$ patients had bone metastases and $36(32.1 \%)$ had lymph node metastases. 81 (72.4\%) patients had an Eastern Cooperative Oncology Group performance status (ECOG-PS) of 0 or 1, with 31 (18.61\%) having an ECOG-PS of 2. Gleason score was available for $112(100 \%)$ patients; $61(54.5 \%)$ patients had a Gleason score of less than 7, and 51 (45.5\%) patients had a score of $8-10$. The baseline PSA at starting AA was 63.4(25.1 200.2) ng/dI. At a median follow-up of 20.2 months, 59 (52.7\%) patients had died. The median OS, rPFS and PSA-PFS were 22.2(20.3 24.1) months, 9.7(9.0 10.4) months and $8.9(7.8 \sim 10.0)$ months respectively.

\section{The pre-albumin level variation during AA treatment in the post-chemotherapy and chemotherapy-naïve MCRPC}

In the post-chemotherapy setting, the serum pre-albumin values of $32 \mathrm{mCRPC}$ patients were $<20 \mathrm{mg} / \mathrm{dL}$ before AA treatment, while after 3 months of AA treatment 41 were $<20 \mathrm{mg} / \mathrm{dL}$. In the chemotherapy-naïve setting, the serum pre-albumin values of $28 \mathrm{mCRPC}$ patients were $<20 \mathrm{mg} / \mathrm{dL}$ before AA treatment, while after 3 months of AA treatment 30 were $<20 \mathrm{mg} / \mathrm{dL}$. During 3 months of AA treatment, $24(34.3 \%)$ of 70 chemotherapy-naïve mCRPC patients showed their serum pre-albumin values decreased, while $24(57.1 \%)$ of 42 postchemotherapy mCRPC patients showed their serum pre-albumin values decreased. Post-chemotherapy mCRPC patients showed serum pre-albumin values decreased more frequently than chemotherapy-naïve patients $(\mathrm{p}=0.029)$.

\section{The prognostic factors evaluation of $\mathrm{MCRPC}$ patients treated with AA}

In relation to pre-albumin value, the median OS, rPFS, PSA PFS were 31.6 months (95\%CI:26.7-36.5) and 18.6 months (95\%CI: 17.0-20.2) ( $\mathrm{p}<0.001), 13.6$ months (95\%CI: 12.3-14.9) and 6.2 months (95\% CI: 4.8-7.6) ( $\mathrm{p}<0.001), 12.1$ months (95\% CI: 11.5-12.7) and 5.7 months (95\%CI: 4.7-6.7) $(\mathrm{p}<0.001)$ in patients with baseline pre-albumin value $\geq 20 \mathrm{mg} / \mathrm{dL}$ or $<20 \mathrm{mg} / \mathrm{dL}$ respectively (Figure 1 ). Based on the values of pre-albumin before and after 3 months of AA treatment, we divided these patients into 4 groups: high-high $(\mathrm{n}=18)$, high-low $(\mathrm{n}=34)$, low-high $(n=22)$ and low-low group $(n=38)$ (Table 5). Highhigh group showed a significantly better OS, PSA PFS, rPFS than the other 3 groups (Figure 2).

Table 1. Clinical characteristics of $m C R P C$ patients in the present study.

\begin{tabular}{|c|c|}
\hline Parameters & Overall $(n=112)$ \\
\hline Age (median, interquartile range), years & $72(66 \sim 77)$ \\
\hline Percentage of Prior Chemotherapy & $42(37.5 \%)$ \\
\hline PSA (median, interquartile range), $\mathrm{ng} / \mathrm{mL}$ & $63.4(25.1 \sim 200.2)$ \\
\hline $\mathrm{LDH}$ (median, interquartile range), $\mathrm{U} / \mathrm{L}$ & 196(155 271.75) \\
\hline \multicolumn{2}{|l|}{ Pre-Alb level at baseline } \\
\hline$\leq 20 \mathrm{mg} / \mathrm{dL}$ & $60(53.6 \%)$ \\
\hline$>20 \mathrm{mg} / \mathrm{dL}$ & $52(46.4 \%)$ \\
\hline \multicolumn{2}{|l|}{ Alb level at baseline } \\
\hline$\leq 35 \mathrm{~g} / \mathrm{L}$ & $13(11.6 \%)$ \\
\hline$>35 \mathrm{~g} / \mathrm{L}$ & $99(88.4 \%)$ \\
\hline \multicolumn{2}{|l|}{ NLR } \\
\hline$\leq 3$ & $75(67 \%)$ \\
\hline$>3$ & $37(33.0 \%)$ \\
\hline \multicolumn{2}{|l|}{ Gleason Score } \\
\hline$\leq 7$ & $61(54.5 \%)$ \\
\hline$>7$ & $51(45.5 \%)$ \\
\hline \multicolumn{2}{|l|}{ Metastatic site } \\
\hline Bone metastasis & $112(100 \%)$ \\
\hline \multicolumn{2}{|l|}{ The number of bone metastasis } \\
\hline$\leq 10$ & $25(22.3 \%)$ \\
\hline$>10$ & $87(77.7 \%)$ \\
\hline Lymph node metastasis & $36(32.1 \%)$ \\
\hline \multicolumn{2}{|l|}{ ECOG score } \\
\hline$\leq 1$ & $61(54.5 \%)$ \\
\hline 2 & $51(45.5 \%)$ \\
\hline Median PSA PFS (median, 95\% CI), months & $8.9(7.8 \sim 10.0)$ \\
\hline Median rPFS (median, 95\% CI), months & $9.7(9.0 \sim 10.4)$ \\
\hline Median OS (median, 95\% CI), months & $22.2(20.3 \sim 24.1)$ \\
\hline Median follow-up (median, interquartile range), months & $20.2(14 \sim 22.9)$ \\
\hline \multicolumn{2}{|c|}{$\begin{array}{l}\text { Abbreviations: ADT, androgen-deprivation therapy; mCRPC, metastatic } \\
\text { castration-resistant prostate cancer; ECOG PS, Eastern Cooperative Oncology } \\
\text { Group performance status; OS, overall survival; rPFS, radiographic } \\
\text { progression-free survival; PSA-PFS, PSA progression-free survival; HR, hazard } \\
\text { ratio; LDH, lactic dehydrogenase; NLR, neutrophil-to-lymphocyte ratio; }\end{array}$} \\
\hline
\end{tabular}

In univariate analysis, 7 variables were significant determinants of the PSA-PFS, rPFS and OS (Table 3). In multivariate analysis, low pre-albumin level (HR, 8.7; $\mathrm{P}<0.001)$ was an independent predictor of PSA-PFS, along with short duration of response to ADT (HR, 8.6; $\mathrm{P}<0.001)$, previous chemotherapy (HR, 2.2; $\mathrm{P}=0.002), \mathrm{LDH} \geq 196 \mathrm{U} / \mathrm{L}(\mathrm{HR}, 3.1 ; \mathrm{P}<0.001)$, ECOG PS $>1$ (HR, 11.3; $\mathrm{P}=0.002)$, but hypoalbuminemia was not. As to rPFS, multivariable analysis also demonstrated that low pre-albumin level (HR, 3.7; $\mathrm{P}=0.003$ ) was an independent predictor of rPFS, along with short duration of response to ADT (HR, 4.3; $\mathrm{P}=0.002)$, previous chemotherapy ( $\mathrm{HR}, 2.2 ; \mathrm{P}=0.002)$, $\mathrm{LDH} \geq 196 \mathrm{U} / \mathrm{L}(\mathrm{HR}, 3.1 ; \mathrm{P}<0.001)$, ECOG PS $>1$ (HR, 4; 
$\mathrm{P}=0.006$ ), but hypoalbuminemia was not. In multivariate analysis, low pre-albumin level (HR, 13.2; $\mathrm{P}<0.001)$, previous chemotherapy $(\mathrm{HR}, 2.5$; $\mathrm{P}=0.01)$ and ECOG PS>1 (HR, 3.1; $\mathrm{P}=0.04)$ remained significant predictors of OS, while hypoalbuminemia was not (Table 4). Furthermore, in multivariate analysis, low pre-albumin level was still a significant independent prognostic predictor not only in the chemotherapy-naïve but also in the postchemotherapy setting separately in this study (data not shown).

Table 2. Clinical characteristics of chemotherapy-naïve and post-chemotherapy mCRPC patients.

\begin{tabular}{llll}
\hline Parameters & $\begin{array}{l}\text { Chemotherapy-naïve } \\
(\mathrm{n}=70)\end{array}$ & $\begin{array}{l}\text { Post-chemotherapy } \\
(\mathrm{n}=42)\end{array}$ & \\
\hline $\begin{array}{l}\text { Age (median, } \\
\text { interquartile range), } \\
\text { years }\end{array}$ & $70.5(65.8 \sim 78)$ & $74.5(65.3-77)$ & 0.8 \\
$\begin{array}{l}\text { Percentage of Prior } \\
\text { Chemotherapy }\end{array}$ & $0(0 \%)$ & $42(100 \%)$ & - \\
$\begin{array}{l}\text { PSA (median, } \\
\text { interquartile range), }\end{array}$ & $52.6(16.5 \sim 200)$ & $68.9(31.9 \sim 206.6)$ & 0.29 \\
$\begin{array}{l}\text { ng/mL } \\
\text { Gleason Score }\end{array}$ & & & \\
$\leq 7$ & $42(60 \%)$ & $19(45.2 \%)$ & 0.13 \\
$>7$ & $28(40 \%)$ & $28(54.8 \%)$ & - \\
$\begin{array}{l}\text { Metastatic site } \\
\text { Bone metastasis }\end{array}$ & $70(100 \%)$ & $42(100 \%)$ & 0.8 \\
$\begin{array}{l}\text { The number of bone } \\
\text { metastasis }\end{array}$ & & & \\
$\leq 10$ & $15(21.4 \%)$ & $21(30 \%)$ & \\
$>10$ & $55(78.6 \%)$ & $49(70 \%)$ & \\
\hline
\end{tabular}

\section{Predictive accuracy of prognostic model of MCRPC treated with AA}

In multivariate analysis, pre-albumin level, previous chemotherapy, ECOG PS $>1$ remained significant predictors of OS. In the present study, we combine these indices to build a prognostic model of
mCRPC treated with AA. The c- index of base model including previous chemotherapy and ECOG PS was 0.814, while it increased to 0.845 when adding pre-albumin level in the model.

\section{Discussion}

Markers of host nutrition, such as pre-albumin, have gained increasing attention as prognostic markers in cancers. The prognostic factor of baseline pre-albumin and pre-albumin change during AA therapy was explored in the present analysis. We investigated baseline pre-albumin level and its change during the first 3 months of AA treatment, and other standard prognostic factors in $112 \mathrm{mCRPC}$ patients treated with AA. Multivariate analysis showed that baseline pre-albumin level was an independent predictor of OS, PSA PFS and rPFS, but not hypoalbuminemia. To the best of our knowledge, this study was the first to investigate the prognostic impact of pre-albumin level in PCA patients.

It is well accepted that nutrition is an important determinant of immune responses and that malnutrition impairs the immune system, suppressing immune functions that are fundamental to host protection [27-29]. Furthermore, protein energy malnutrition is associated with significant immunodeficiency, especially in the cell-mediated mechanisms, which is crucial in the host defenses against infection or cancer [29]. Although early detection and prevention of malnutrition is significant, it is frequently underdiagnosed or overlooked during anti-cancer treatment, making it necessary to evaluate cancer patients with malnutrition. These findings support the importance of the role of nutritional markers in MCRPC and the necessity to investigate more accurate nutritional indices to predict prognosis in MCRPC treated with AA.
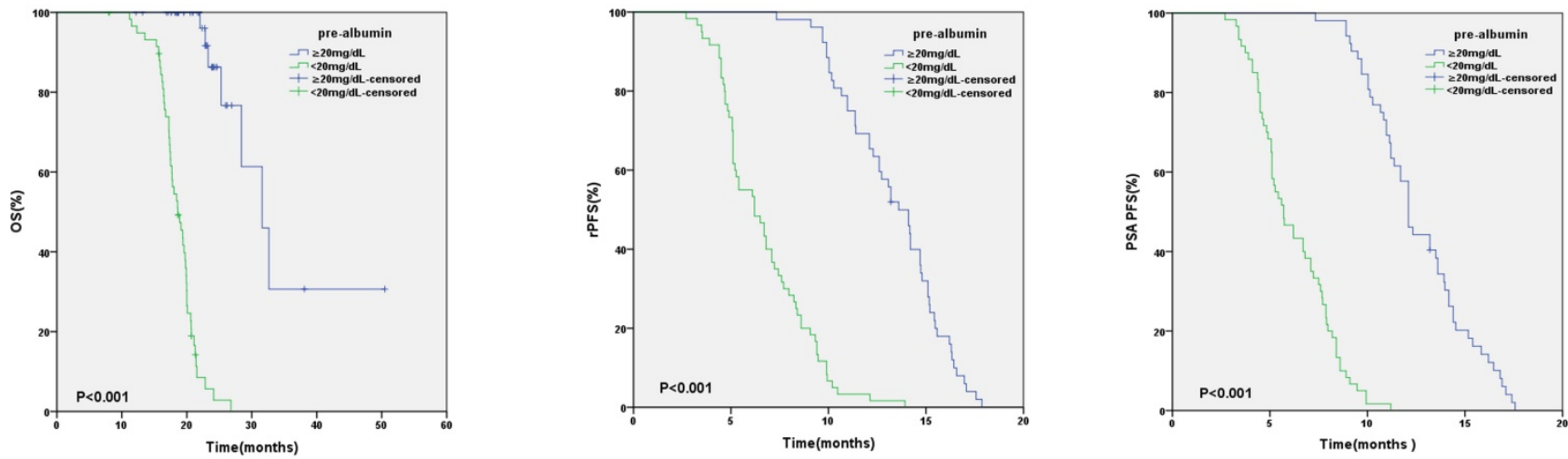

Figure 1. Kaplan-Meier analysis based on baseline pre-albumin value 

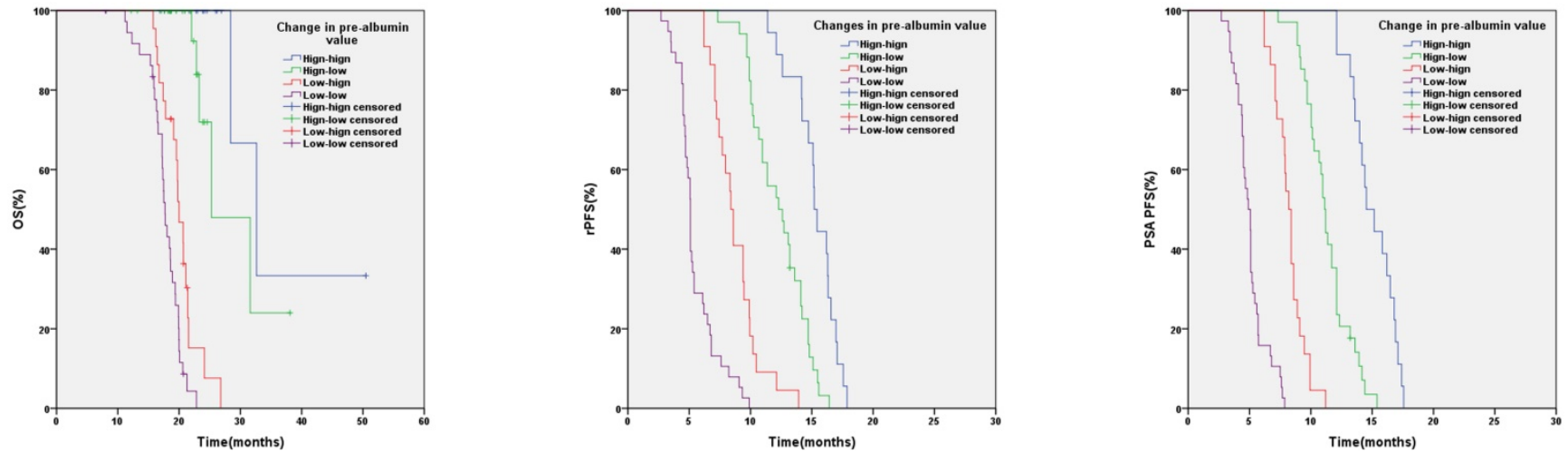

Figure 2. Kaplan-Meier analysis based on pre-albumin value at baseline and after 3 months of AA

Table 3. Univariable analyses of various clinical parameters in mCRPC

\begin{tabular}{|c|c|c|c|c|c|c|}
\hline \multirow[t]{2}{*}{ Parameters } & \multicolumn{2}{|l|}{ PSA PFS } & \multicolumn{2}{|l|}{ OS } & \multicolumn{2}{|l|}{ rPFS } \\
\hline & HR (95\% CI) & P-value & HR $(95 \% \mathrm{CI})$ & P-value & HR $(95 \%$ CI) & P-value \\
\hline Age (years) & $1(0.98-1.02)$ & 0.8 & $1(0.97-1.04)$ & 0.8 & $1(0.99 \sim 1.04)$ & 0.4 \\
\hline PSA $(<62 \mu \mathrm{g} / \mathrm{L}$ VS $>62 \mu \mathrm{g} / \mathrm{L})$ & $1.1(0.8-1.7)$ & 0.5 & $1(0.6-1.6)$ & 0.9 & $1.1(0.8-1.7)$ & 0.5 \\
\hline Previous chemotherapy (yes vs no) & $2.1(1.4-3.1)$ & 0.001 & $5.9(3-11.8)$ & $<0.001$ & $1.9(1.2-2.8)$ & 0.002 \\
\hline NLR $(\geq 3$ VS $<3)$ & $30(14.8-60.9)$ & $<0.001$ & $13.6(6.8-27.2)$ & $<0.001$ & $19.5(10.7-35.8)$ & $<0.001$ \\
\hline Gleason Score ( $\leq 7$ VS >7) & $1.1(0.8-1.7)$ & 0.5 & $1.3(0.8-2.1)$ & 0.4 & $1.1(0.8-1.6)$ & 0.6 \\
\hline LDH $(\geq 196 \mathrm{U} / \mathrm{L}$ VS <196 U/L) & $3.5(2.3-5.3)$ & $<0.001$ & $3.7(2-6.8)$ & $<0.001$ & $3.5(2.3-5.3)$ & $<0.001$ \\
\hline Pre-Alb $(<20 \mathrm{mg} / \mathrm{dL}$ VS $\geq 20 \mathrm{mg} / \mathrm{dL})$ & $17(9.3-31.3)$ & $<0.001$ & $30.5(10.7-86.5)$ & $<0.001$ & $11.4(6.7-19.3)$ & $<0.001$ \\
\hline $\mathrm{Alb}(<35 \mathrm{~g} / \mathrm{L} \mathrm{VS} \geq 35 \mathrm{~g} / \mathrm{L})$ & $2.2(1.2-4.1)$ & 0.007 & $3(1.5-6.1)$ & 0.002 & $2.7(1.5-4.9)$ & 0.001 \\
\hline $\begin{array}{l}\text { The duration of response to ADT } \\
(<12 \text { months VS } \geq 12 \text { months) }\end{array}$ & $75.3(28.3-200)$ & $<0.001$ & $11.4(5.9-22)$ & $<0.001$ & $35.5(16.9-74.6)$ & $<0.001$ \\
\hline ECOG PS (<2 VS 2) & 28.6(14.1-57.8) & $<0.001$ & $16.9(7.6-37.4)$ & $<0.001$ & $19.3(9.7 \sim 38)$ & $<0.001$ \\
\hline
\end{tabular}

Abbreviations: ADT, androgen-deprivation therapy; mCRPC, metastatic castration-resistant prostate cancer; ECOG PS, Eastern Cooperative Oncology Group performance status; OS, overall survival; rPFS, radiographic progression-free survival; PSA-PFS , PSA progression-free survival; HR, hazard ratio; LDH, lactic dehydrogenase; NLR, neutrophil-to-lymphocyte ratio; Alb, albumin.

Table 4. Multivariable analyses of various clinical parameters in mCRPC

\begin{tabular}{|c|c|c|c|c|c|c|}
\hline \multirow[t]{2}{*}{ Parameters } & \multicolumn{2}{|l|}{ PSA PFS } & \multicolumn{2}{|l|}{ OS } & \multicolumn{2}{|l|}{ rPFS } \\
\hline & HR $(95 \% \mathrm{CI})$ & P-value & HR $(95 \%$ CI $)$ & P-value & HR $(95 \%$ CI) & P-value \\
\hline Previous chemotherapy (yes vs no) & $2.4(1.4-4.0)$ & 0.001 & $2.5(1.2-5.4)$ & 0.01 & $2.2(1.3-3.7)$ & 0.002 \\
\hline $\operatorname{NLR}(\geq 3$ VS <3) & $2.4(0.9-6.1)$ & 0.07 & $2(0.9-4.5)$ & 0.4 & $1.9(0.9-4.5)$ & 0.1 \\
\hline LDH $(\geq 196 \mathrm{U} / \mathrm{L}$ VS $<196 \mathrm{U} / \mathrm{L})$ & $3.1(1.8-5.5)$ & $<0.001$ & $1.7(0.7-4)$ & 0.2 & $3.1(1.8-5.5)$ & $<0.001$ \\
\hline Pre-Alb $(<20 \mathrm{mg} / \mathrm{dL} \mathrm{VS} \geq 20 \mathrm{mg} / \mathrm{dL})$ & $8.7(3.6-20.7)$ & $<0.001$ & $13.2(4-43.9)$ & $<0.001$ & $3.7(1.6-8.6)$ & 0.003 \\
\hline $\mathrm{Alb}(<35 \mathrm{~g} / \mathrm{L} \mathrm{VS} \geq 35 \mathrm{~g} / \mathrm{L})$ & $1.3(0.7-2.6)$ & 0.3 & $1.1(0.5-2.4)$ & 0.9 & $1.3(0.7-2.4)$ & 0.5 \\
\hline $\begin{array}{l}\text { The duration of response to ADT } \\
(<12 \text { months } V S \geq 12 \text { months })\end{array}$ & $8.6(2.7-27.7)$ & $<0.001$ & $0.9(0.2-4.1)$ & 0.9 & $4.3(1.7-11.3)$ & 0.002 \\
\hline ECOG PS (2 VS <2) & $11.3(4.4-28.7)$ & 0.002 & $3.1(1.0-8.9)$ & 0.04 & $4(1.5 \sim 10.7)$ & 0.006 \\
\hline
\end{tabular}

Abbreviations: ADT, androgen-deprivation therapy; mCRPC, metastatic castration-resistant prostate cancer; ECOG PS, Eastern Cooperative Oncology Group performance status; OS, overall survival; rPFS, radiographic progression-free survival; PSA-PFS , PSA progression-free survival; HR, hazard ratio; LDH, lactic dehydrogenase; NLR, neutrophil-to-lymphocyte ratio.

Table 5. Changes in pre-albumin value and clinical outcome.

\begin{tabular}{|c|c|c|c|c|c|c|}
\hline \multirow[t]{2}{*}{ Pre-albumin } & \multicolumn{2}{|l|}{ PSA PFS } & \multicolumn{2}{|l|}{ rPFS } & \multicolumn{2}{|l|}{ OS } \\
\hline & Median PSA PFS(95\% CI) & $\begin{array}{l}\text { P-value; } \\
\text { HR }\end{array}$ & Median rPFS (95\% CI) & $\begin{array}{l}\text { P-value; } \\
\text { HR }\end{array}$ & Median OS (95\% CI) & $\begin{array}{l}\text { P-value; } \\
\text { HR }\end{array}$ \\
\hline Hign-hign & 14.5(12.9-16.1) & & 15.2(14.7-15.7) & & $32.6(25.9-39.4)$ & \\
\hline Hign-low & 11.1(10.7-11.6) & $\begin{array}{l}<0.001 ; \\
26.2\end{array}$ & 12.2(10.4-14.2) & $\begin{array}{l}<0.001 \\
17.4\end{array}$ & $25.3(17.9-32.7)$ & $\begin{array}{l}0.048 ; \\
2.4\end{array}$ \\
\hline Low-hign & $8.2(7.7-8.7)$ & $\begin{array}{l}<0.001 ; \\
42.7\end{array}$ & $8.4(7.7-9.1)$ & $\begin{array}{l}<0.001 ; \\
39.3\end{array}$ & $20.0(18.7-21.3)$ & $\begin{array}{l}<0.001 \\
27.6\end{array}$ \\
\hline Low-low & $4.9(4.3-5.5)$ & $\begin{array}{l}<0.001 \\
48.7\end{array}$ & 5.1(4.9-5.3) & $\begin{array}{l}<0.001 \\
48.7\end{array}$ & $17.7(16.9-18.5)$ & $\begin{array}{l}<0.001 \\
38.9\end{array}$ \\
\hline
\end{tabular}


Few studies have been conducted to determine the influence of pretreatment nutritional status in the prognosis of mCRPC. Pre-albumin is a visceral liver-synthesized protein, which is sensitive to determine malnutrition, and its biological half-life is approximately 2.5 days. In addition, it is not altered by stress or acute inflammation [30]. Albumin cannot be a suitable sensitive indicator of nutritional insufficiency as it has a half-life of 20 days [31]. In addition, their levels are significantly influenced with age and inflammation compared with pre-albumin $[32,33]$. So we hypothesized that the pre-albumin level may be a better indicator than albumin level for determining nutritional status and prognosis of mCRPC. In our study, low pre-albumin level was an independent predictor in mCRPC patients treated with AA but not hypoalbuminemia in multivariable analysis.

It is interesting to note that in our study we found high- high group showed a significantly better OS, PSA PFS, rPFS than other group. Compared with high-low group, high- high group showed a significantly better OS, PSA PFS, rPFS. Furthermore, compared with low-low group, low-high group showed a significantly better clinical outcome as well. Owing to these, we consumed that lower serum pre-albumin level may indicate worse prognosis of mCRPC patients treated with AA and serial pre-albumin evaluation would help clinicians in selecting appropriate therapy regimen timely. These may strength the predictor role of pre-albumin in mCRPC. Moreover, these may indicate that if serum pre-albumin value is low, nutritional support or supplementation could improve the prognosis of mCRPC patients treated with AA.

In multivariate analysis, previous chemotherapy was an adverse prognostic factor in MCRPC patients treated with AA in our study. Furthermore, in the present study, during 3 months of AA treatment post-chemotherapy mCRPC patients showed serum pre-albumin values decreased more frequently than chemotherapy-naïve patients $(p=0.029)$. It might showed that previous chemotherapy could lead to serum pre-albumin values decrease, which indicated adverse prognosis in mCRPC patients treated with AA. This phenomenon may partly account for why post-chemotherapy mCRPC patients showed worse prognosis than chemotherapy-naïve patients. In the future, to determine this conclusion, we would conduct further research to determine whether any clinical factors led to pre-albumin variation after AA treatment.

Because the most widely used routine prognostic assessment of mCRPC currently still relies on traditional clinicopathological prognostic variables including LDH, NLR, the type and duration of prior therapy [34-36], recent progress in the identification of genetic and molecular alterations in $\mathrm{MCRPC}$ has been made [37-39]. Although the predictive accuracy of prognostic model might be enhanced by these biomarkers, the high costs of analysis, the time-consuming preparation and the lack of evidence together prevent them into clinical practice. In the present study, we showed that adding pre-albumin was able to raise the predictive accuracy in this cohort of $\mathrm{mCRPC}$ regarding to OS. The c-index of the model with traditional variables plus pre-albumin became higher than the base model alone in OS (0.845 vs. 0.814 ) in our cohort of patients. Because of the plentiful treatment choices in $\mathrm{MCRPC}$ and high cost of mCRPC treatment, detailed understanding of individual risk factor associated with patients' prognosis will impart benefits for clinical outcome and individual patient treatment choices, such as chemotherapy or radiotherapy. Furthermore, the pre-albumin could represent a novel predictive marker of clinical outcome to other treatments such as chemotherapy, radiotherapy in PCA, and should be tested in these clinical situations.

Our results were consistent with previous studies. McKay et al. [35] identified $161 \mathrm{mCRPC}$ patients who had received AA. They concluded duration of primary ADT and no use of prior chemotherapy were significant prognostic factors in mCRPC treated with AA. Chi KN et al identified 762 mCRPC patients treated AA after docetaxel and developed a prognostic index model. They concluded LDH, ECOG PS, presence of liver metastases, albumin, alkaline phosphatase and time from start of initial ADT to start of AA treatment $\leq 36$ months were significant prognostic factors in mCRPC treated with AA after docetaxel. However, this prognostic index model was only suitable for post-chemotherapy mCRPC patients treated with AA.

In contrast to these studies, to our knowledge ours is the first designed to evaluate the impact of nutritional status on survival in mCRPC. Compared with percentage weight loss and BMI to assess pretreatment nutritional status, serum pre-albumin is a more direct, objective, easily measured value. In addition, we developed a new prognostic model for mCRPC treated with AA both in chemotherapy-naïve and post-chemotherapy setting. Furthermore, our results showed serial pre-albumin evaluation might guide clinicians to conduct nutritional support or supplementation to improve the prognosis of mCRPC patients treated with AA and help clinicians change therapy regimen timely.

There were some limitations in our study. Firstly, the relative shorter follow-up might limit the 
accuracy of our results. Secondly, this study was based on the retrospective nature of the data collection and completed at a single center. Additionally, we did not compare the pre-albumin with other nutritional tools. Thirdly, the patients in our study were included with chemotherapy-naïve and post- chemotherapy setting. However, in this study, there was no significant heterogeneity between chemotherapy-naïve and post-chemotherapy setting (table 2). In addition, in separate analysis, these results have also shown serum pre-albumin was a prognostic factor of mCRPC patients treated with AA not only in the chemotherapy-naïve but also in the post- chemotherapy setting separately in this study. Owing to these, the prognosticate factor of serum pre-albumin seemed to be solid in spite of combining chemotherapy-naïve and post- chemotherapy mCRPC for analysis. Further large-scale population-based prospective studies will be needed to conduct to fully consolidate the results.

\section{Implications for Practice}

This prospective cohort study of 112 mCRPC patients showed that serum pre-albumin was an independent prognostic indicator of OS and PFS of mCRPC patients treated with AA. Serial pre-albumin evaluation might guide clinicians to conduct nutritional support or supplementation to improve the prognosis of mCRPC patients treated with AA and help clinicians change therapy regimen timely.

\section{Acknowledgements}

This study was supported by National Natural Science Foundation of China (81572536, 81672850), Science and Technology Commission of Shanghai Municipality (14140901700, 16411969800), the Joint Research Foundation for Innovative Medical Technology of Shanghai Shenkang Hospital Development Center (SHDC12015125), Shanghai Municipal Education Commission (15ZZ058), Shanghai Municipal Commission of Health and Family Planning (201640247), Shanghai Municipal Education Commission-Gaofeng Clinical Medicine Grant Support (20152215), Key Disciplines Group Construction Project of Pudong Health Bureau of Shanghai (PWZxq2014-05), Innovation Fund for Translational Research of Shanghai Jiao Tong University School of Medicine (15ZH4002), and Incubating Program for clinical Research and Innovation of Renji Hospital Shanghai Jiao Tong University School of Medicine (PYZY16-008, PYXJS16-015 ).

\section{Author Contributions}

Conception and Design: FLC, CCC, GSW.
Provision of Study Material or Patients: FLC, CCC, GSW, WYQ, CW, SXG, XF, SGX.

Collection and Assembly of Data: XZX, HJN, ZYJ, PJH, XSW.

Data Analysis and Interpretation: FLC, WR, ZLX, DBJ, XW.

Manuscript Writing: FLC, DBJ.

Final Approval of Manuscript: All the authors.

E-mail addresses:

xuewei@renji.com (Wei, X), dongbaijun@renji.com (Baijun, D), 787371380@qq.com (Liancheng, F), ccf19881216@yeah.net (Chenfei, C), guosanwei@yeah.net (Sanwei, G), iwangyq@163.com (Yanqing, W) caiwen@hotmail.com (Wen, C), shaoxgg@163.com (Xiaoguang, S), xusiran1990@163.com (Fan, X), jiahua.pan@outlook.com (Jiahua, P), yinjiezhu@outlook.com (Yinjie, Z) sgxsean@163.com (Xun, S, X), xiaoxin973@hotmail.com (Zhixiang, $X)$, 591013059@qq.com (Jianian, H), shaoweixie@yeah.net (Shaowei, X), wangruiwr2015@163.com (Rui, W) zhou_li_xin@hotmail.com (Lixin, Z), qianhy100@163.com (Hongyang, Q).

\section{Competing Interests}

The authors have declared that no competing interest exists.

\section{References}

[1] Siegel RL, Miller KD, Jemal A. Cancer statistics, 2016. CA: a cancer journal for clinicians. 2016; 66:7-30

[2] Ryan CJ, Smith MR, Fizazi K, et al. Abiraterone acetate plus prednisone versus placebo plus prednisone in chemotherapy-naive men with metastatic castration-resistant prostate cancer (COU-AA-302): final overall survival analysis of a randomised, double-blind, placebo-controlled phase 3 study. The Lancet Oncology. 2015; 16:152-60

[3] Beer TM, Armstrong AJ, Rathkopf DE, et al. Enzalutamide in metastatic prostate cancer before chemotherapy. The New England journal of medicine. $2014 ; 371: 424-33$

[4] Scher HI, Fizazi K, Saad F, et al. Increased survival with enzalutamide in prostate cancer after chemotherapy. The New England journal of medicine. 2012; 367:1187-97

[5] Basch E, Autio K, Ryan CJ, et al. Abiraterone acetate plus prednisone versus prednisone alone in chemotherapy-naive men with metastatic castration-resistant prostate cancer: patient-reported outcome results of a randomised phase 3 trial. The Lancet Oncology. 2013; 14:1193-9

[6] Ryan CJ, Smith MR, de Bono JS, et al. Abiraterone in metastatic prostate cancer without previous chemotherapy. The New England journal of medicine. 2013; 368:138-48

[7] Rathkopf DE, Smith MR, de Bono JS, et al. Updated interim efficacy analysis and long-term safety of abiraterone acetate in metastatic castration-resistant prostate cancer patients without prior chemotherapy (COU-AA-302). European urology. 2014; 66:815-25

[8] de Bono JS, Logothetis CJ, Molina A, et al. Abiraterone and increased survival in metastatic prostate cancer. The New England journal of medicine. 2011; 364:1995-2005

[9] de Bono JS, Oudard S, Ozguroglu M, et al. Prednisone plus cabazitaxel or mitoxantrone for metastatic castration-resistant prostate cancer progressing after docetaxel treatment: a randomised open-label trial. Lancet. 2010; 376:1147-54

[10] Fizazi K, Scher HI, Molina A, et al. Abiraterone acetate for treatment of metastatic castration-resistant prostate cancer: final overall survival analysis of 
the COU-AA-301 randomised, double-blind, placebo-controlled phase 3 study. The Lancet Oncology. 2012; 13:983-92

[11] Parker C, Nilsson S, Heinrich D, et al. Alpha emitter radium-223 and survival in metastatic prostate cancer. The New England journal of medicine. 2013; 369:213-23

[12] Hutcheson J, Balaji U, Porembka MR, et al. Immunologic and Metabolic Features of Pancreatic Ductal Adenocarcinoma Define Prognostic Subtypes of Disease. Clinical cancer research: an official journal of the American Association for Cancer Research. 2016; 22:3606-17

[13] Sanchez-Lara K, Turcott JG, Juarez E, et al. Association of nutrition parameters including bioelectrical impedance and systemic inflammatory response with quality of life and prognosis in patients with advanced non-small-cell lung cancer: a prospective study. Nutrition and cancer. 2012; 64:526-34

[14] Nuhn P, Vaghasia AM, Goyal J, et al. Association of pretreatment neutrophil-to-lymphocyte ratio (NLR) and overall survival (OS) in patients with metastatic castration-resistant prostate cancer (mCRPC) treated with first-line docetaxel. BJU international. 2014; 114:11-7

[15] Leibowitz-Amit R, Templeton AJ, Omlin A, et al. Clinical variables associated with PSA response to abiraterone acetate in patients with metastatic castration-resistant prostate cancer. Annals of oncology: official journal of the European Society for Medical Oncology / ESMO. 2014; 25:657-62

[16] Lorente D, Mateo J, Templeton AJ, et al. Baseline neutrophil-lymphocyte ratio (NLR) is associated with survival and response to treatment with second-line chemotherapy for advanced prostate cancer independent of baseline steroid use. Annals of oncology: official journal of the European Society for Medical Oncology / ESMO. 2015; 26:750-5

[17] Conteduca V, Crabb SJ, Jones RJ, et al. Persistent Neutrophil to Lymphocyte Ratio $>3$ during Treatment with Enzalutamide and Clinical Outcome in Patients with Castration-Resistant Prostate Cancer. PloS one. 2016; 11:e0158952

[18] Morgan TM, Tang D, Stratton KL, et al. Preoperative nutritional status is an important predictor of survival in patients undergoing surgery for renal cell carcinoma. European urology. 2011; 59:923-8

[19] Fiala O, Pesek M, Finek J, et al. Serum albumin is a strong predictor of survival in patients with advanced-stage non-small cell lung cancer treated with erlotinib. Neoplasma. 2016; 63:471-6

[20] Sejima T, Iwamoto H, Masago T, et al. Low pre-operative levels of serum albumin predict lymph node metastases and ultimately correlate with a biochemical recurrence of prostate cancer in radical prostatectomy patients. Central European journal of urology. 2013; 66:126-32

[21] Huang J, Wang Y, Yuan Y, et al. Preoperative serum pre-albumin as an independent prognostic indicator in patients with localized upper tract urothelial carcinoma after radical nephroureterectomy. Oncotarget. 2017; 8:36772-9

[22] Cai W, Kong W, Dong B, et al. Pretreatment Serum Prealbumin as an Independent Prognostic Indicator in Patients with Metastatic Renal Cell Carcinoma Using Tyrosine Kinase Inhibitors as First-Line Target Therapy. Clinical genitourinary cancer. 2017; 15:437-46

[23] Kelly P, Paulin F, Lamont D, et al. Pre-treatment plasma proteomic markers associated with survival in oesophageal cancer. British journal of cancer. 2012; 106:955-61

[24] Kawai H, Ota H. Low perioperative serum prealbumin predicts early recurrence after curative pulmonary resection for non-small-cell lung cancer. World journal of surgery. 2012; 36:2853-7

[25] Scher HI, Halabi S, Tannock I, et al. Design and end points of clinical trials for patients with progressive prostate cancer and castrate levels of testosterone: recommendations of the Prostate Cancer Clinical Trials Working Group. Journal of clinical oncology: official journal of the American Society of Clinical Oncology. 2008; 26:1148-59

[26] Grass F, Bertrand PC, Schafer M, et al. Compliance with preoperative oral nutritional supplements in patients at nutritional risk--only a question of will? European journal of clinical nutrition. 2015; 69:525-9

[27] Chandra RK, Kumari S. Nutrition and immunity: an overview. The Journal of nutrition. 1994; 124:1433S-5S

[28] Marcos A, Nova E, Montero A. Changes in the immune system are conditioned by nutrition. European journal of clinical nutrition. 2003; 57 (Suppl 1):66-9

[29] Chandra RK. Nutrition and the immune system: an introduction. The American journal of clinical nutrition. 1997; 66:460-3(S)

[30] Myron Johnson A, Merlini G, Sheldon J, Ichihara K, Scientific Division Committee on Plasma Proteins IFoCC, Laboratory M. Clinical indications for plasma protein assays: transthyretin (prealbumin) in inflammation and malnutrition. Clinical chemistry and laboratory medicine. 2007; 45:419-26

[31] Gregg JR, Cookson MS, Phillips S, et al. Effect of preoperative nutritional deficiency on mortality after radical cystectomy for bladder cancer. The Journal of urology. 2011; 185:90-6

[32] Shenkin A. Serum prealbumin: Is it a marker of nutritional status or of risk of malnutrition? Clinical chemistry. 2006; 52:2177-9

[33] Cano N. Hemodialysis, inflammation and malnutrition. Nefrologia: publicacion oficial de la Sociedad Espanola Nefrologia. 2001; 21:437-42

[34] Lolli C, Caffo O, Scarpi E, et al. Systemic Immune-Inflammation Index Predicts the Clinical Outcome in Patients with mCRPC Treated with Abiraterone. Frontiers in pharmacology. 2016; 7:376.

[35] McKay RR, Werner L, Fiorillo M, Nakabayashi M, Kantoff PW, Taplin ME. Predictors of duration of abiraterone acetate in men with castration-resistant prostate cancer. Prostate cancer and prostatic diseases. 2016; 19:398-405.
[36] Fan L, Wang X, Chi C, et al. Prognostic nutritional index predicts initial response to treatment and prognosis in metastatic castration-resistant prostate cancer patients treated with abiraterone. The Prostate. 2017; 77:1233-41

[37] Seitz AK, Thoene S, Bietenbeck A, et al. AR-V7 in Peripheral Whole Blood of Patients with Castration-resistant Prostate Cancer: Association with Treatment-specific Outcome Under Abiraterone and Enzalutamide. European urology. 2017; 14:

[38] Lorente D, Olmos D, Mateo J, et al. Decline in Circulating Tumor Cell Count and Treatment Outcome in Advanced Prostate Cancer. European urology. 2016; 70:985-92

[39] Fan L, Wang Y, Chi C, et al. Chromogranin A and neurone-specific enolase variations during the first 3 months of abiraterone therapy predict outcomes in patients with metastatic castration-resistant prostate cancer. BJU international. $2017 ; 120: 226-32$ 